\title{
Estrogen-related receptor $\gamma$ is upregulated in liver cancer and its inhibition suppresses liver cancer cell proliferation via induction of p21 and p27
}

\author{
Ji-Hyun Kim ${ }^{1,2,7}$, Yeon-Kyung Choi ${ }^{1,3,7}$, Jun-Kyu Byun ${ }^{3,7}$, Mi-Kyung Kim ${ }^{4}$, Yu Na Kang ${ }^{5}$, Seong Heon Kim ${ }^{6}$, \\ Sungwoo Lee ${ }^{6}$, Byoung Kuk Jang ${ }^{4}$ and Keun-Gyu Park ${ }^{1,2,3}$
}

\begin{abstract}
Orphan nuclear receptor estrogen-related receptor $\gamma(E R R \gamma)$ regulates cell growth and tumorigenesis in various cancers. However, the clinical relevance of ERR $\gamma$ to hepatocellular carcinoma (HCC) remains unclear. Here we examined the clinical significance of ERR $\gamma$ in HCC and its potential as a therapeutic target. ERR $\gamma$ levels in tissues from completely resected specimens from 190 HCC patients were examined immunohistochemically and their association with clinical stage and pathological grade was analyzed. Small interfering RNA (siRNA)-mediated knockdown of ERR $\gamma$ (siRNA-ERR $\gamma$ ) or an ERR $\gamma$ inverse agonist, GSK5182, were also used to examine the effects of ERR $\gamma$ inhibition on the proliferation and growth of a human hepatoma cell line, PLC/PRF/5. Immunohistochemical analysis revealed that tumor tissues showed higher levels of ERR $\gamma$-positivity than adjacent non-tumor lesions. Tumors showing high levels of ERR $\gamma$ immunoreactivity also had advanced tumor node metastasis (TNM) and Barcelona Clinic Liver Cancer stages and a higher Edmondson-Steiner grade. In addition, high-level expression of ERR $\gamma$ in tumors of advanced TNM stage correlated with poorer overall survival. Treatment of PLC/PRF/5 cells with siRNA-ERR $\gamma$ or GSK5182 inhibited proliferation through G1 arrest, increased expression of p21 and p27 and decreased expression of phosphorylated retinoblastoma protein. GSK5182-induced reactive oxygen species also suppressed the proliferation of PLC/PRF/5 cells. The present study showed that ERR $\gamma$ expression is clinically significant in HCC; therefore, it can be considered a biomarker for HCC diagnosis. Moreover, the results provide a rationale for the use of ERR $\gamma$ inhibitors such as GSK5182 as potential therapeutic agents.

Experimental \& Molecular Medicine (2016) 48, e213; doi:10.1038/emm.2015.115; published online 4 March 2016
\end{abstract}

\section{INTRODUCTION}

Hepatocellular carcinoma (HCC) is the sixth most common and leading cause of cancer-related death worldwide. ${ }^{1}$ Curative treatment is effective only if the cancer is diagnosed at an early stage; however, recurrence rates are high and long-term survival is poor. ${ }^{2}$ Therefore, it is imperative to identify markers that can improve early diagnostic accuracy and predict disease prognosis; such markers would enable clinicians to decide the most appropriate course of treatment. Increased understanding of the signaling pathways underpinning HCC development and progression has stimulated interest in the identification of molecular biomarkers that may predict the prognosis of patients with HCC and/or be developed as potential therapeutic targets. ${ }^{3}$

Estrogen receptor (ER)-related receptors (ERRs; ERR $\alpha$, ERR $\beta$, and ERR $\gamma$ ) belong to the NR3B group of nuclear receptors and are closely related to ERs, sharing highly homologous DNA-binding domains; however, they are constitutively active and do not bind to natural estrogen. ${ }^{4,5}$ Among these receptors, ERR $\gamma$ is expressed in tissues associated with high metabolic demand, including heart, skeletal muscle and brown adipose tissue. ${ }^{6}$ Accumulating evidence indicates that $\mathrm{ERR} \gamma$ is involved in controlling mitochondrial biogenesis and

\footnotetext{
${ }^{1}$ Department of Internal Medicine, Kyungpook National University School of Medicine, Daegu, Republic of Korea; ${ }^{2}$ Research Institute of Aging and Metabolism, Kyungpook National University School of Medicine, Daegu, Republic of Korea; ${ }^{3}$ Leading-edge Research Center for Drug Discovery and Development for Diabetes and Metabolic Disease, Kyungpook National University Hospital, Daegu, Republic of Korea; ${ }^{4}$ Department of Internal Medicine, Keimyung University School of Medicine, Daegu, Republic of Korea; ${ }^{5}$ Department of Pathology, Keimyung University School of Medicine, Daegu, Republic of Korea and ${ }^{6}$ New Drug Development Center, Daegu-Gyeongbuk Medical Innovation Foundation, Daegu, Republic of Korea

${ }^{7}$ These authors contributed equally to this work.

Correspondence: Professor BK Jang, Department of Internal Medicine, Keimyung University School of Medicine, 56 Dalseong-ro, Jung-Gu, Daegu 41931 , Republic of Korea.

E-mail: jangha106@dsmc.or.kr

or Professor K-G Park, Department of Internal Medicine, Kyungpook National University School of Medicine, 130 Dongdeok-ro, Jung-gu, Daegu 41944 , Republic of Korea.

E-mail: kpark@knu.ac.kr

Received 21 October 2015; revised 11 November 2015; accepted 18 November 2015
} 
cellular energy homeostasis and that it has a pivotal role in metabolic deregulation in various tissues. ${ }^{7}$ Besides its role in metabolic disease, recent studies report the clinical significance of ERR $\gamma$ in estrogen-dependent cancers such as those of the breast and endometrium. For example, ERR $\gamma$ is overexpressed in breast cancer and correlates with lymph node-positive status, indicating that ERR $\gamma$ has an important role in promoting the growth and metastasis of breast cancer. ${ }^{8,9}$ ERR $\gamma$ is upregulated during the acquisition of tamoxifen resistance, suggesting that it may be a predictive marker for a poor therapeutic response by breast cancer. ${ }^{8,9}$ Similarly, ERR $\gamma$ is upregulated in endometrial cancer, and its expression increases with clinical stage, depth of myometrial invasion and an increase in the number of metastatic lymph nodes. ${ }^{10}$ Moreover, ERR $\gamma$ mediates estrogeninduced proliferation of endometrial cancer cells. ${ }^{11}$ However, no studies have examined the clinical relevance of ERR $\gamma$ to HCC or its potential as a therapeutic target.

Aberrant expression of cell cycle protein kinases is a hallmark of human malignancy; therefore, a considerable number of small molecules targeting cell cycle regulatory proteins have been studied as candidate treatments for attenuating the proliferation of cancer cells. ${ }^{12}$ Cyclindependent kinase inhibitors such as $\mathrm{p} 21^{\mathrm{WAF} 1}$ and $\mathrm{p} 27^{\mathrm{Kip} 1}$ are negative regulators of the cell cycle check point and essential mediators of cell cycle arrest. ${ }^{13,14}$ Therefore, increasing the expression of p21 or p27 has been attempted to induce growth arrest in cancer cells. ${ }^{15}$ In this context, expression of the ERR subfamily in tumor tissues has focused attention on their role in cancer cell cycle progression in conjunction with regulation of cyclin-dependent kinase inhibitor. ${ }^{16}$ Upregulation of ERR $\alpha$ in breast and colon cancer has a role in cell cycle progression, ${ }^{17,18}$ whereas downregulating ERR $\beta$ affects the cell cycle and tumor progression in prostate cancer. ${ }^{19,20}$ Accordingly, small molecules that enable the selective inhibition of ERR $\alpha$ or activate ERR $\beta$ induce cell cycle arrest and inhibit cell proliferation. For example, a synthetic ERR $\alpha$ inverse agonist, XCT790, downregulates cell proliferation and inhibits G1- to S-phase transition by upregulating $\mathrm{p} 21 .^{21}$ On the other hand, the ERR $\beta$ agonist, DY131, inhibits the ERR $\beta$-induced growth of prostate cancer cells, which is attributed to p21 induction. ${ }^{20}$ However, despite the increasing attention focused on the relevance of ERR $\gamma$ in cancer, no studies have examined whether the ERR $\gamma$ inverse agonist, GSK5182, effectively modulates cell cycle progression.

Therefore, the present study examined the expression of ERR $\gamma$ in HCC and compared it with that in adjacent nontumor lesions. We also investigated the correlation between ERR $\gamma$ expression and clinicopathological stage. Furthermore, we determined whether GSK5182 inhibits cell cycle progression and HCC cell proliferation.

\section{MATERIALS AND METHODS}

\section{Patients and specimens}

Samples of HCC and adjacent non-tumor tissues were obtained from 190 HCC patients who underwent surgical resection at Keimyung University Dongsan Hospital in Daegu, Korea from 2001 to 2011.
Patients who had received preoperative anticancer treatment, such as transarterial chemoembolization or local ablation therapy, were excluded. Tumor staging was determined according to the American Joint Committee on Cancer (AJCC 2010, Seventh Edition) tumor node metastasis (TNM) staging system ${ }^{22}$ and the Barcelona Clinic Liver Cancer (BCLC) staging system for HCC. ${ }^{23}$ Tumor differentiation was defined according to the Edmondson grading system.

\section{Immunohistochemical staining and scoring}

A tissue microarray containing tissue from HCC $(n=190)$ and adjacent non-tumor tissues $(n=187)$ was constructed as previously described, ${ }^{24}$ Immunohistochemical analysis was performed on $5-\mu \mathrm{m}$ thick tissue sections using an anti-ERR $\gamma$ antibody (1:1600) (R\&D Systems, Minneapolis, MN, USA). ERR $\gamma$ expression was evaluated using the proportion score, defined as the estimated fraction of positively stained tumor cells $(0$, none; $1+,<10 \% ; 2+, 10-25 \%$; $3+$, $>25 \%$ ). Scores of 2 and 3 were considered as high-level expression. All slides were evaluated independently three times by a senior pathologist blinded to the patients' clinical information and follow-up data.

\section{Cell culture}

The human hepatoma cell line PLC/PRF/5 was purchased from ATCC (American Type Culture Collection, Manassas, VA, USA) and grown at $37^{\circ} \mathrm{C} / 5 \% \mathrm{CO}_{2}$ in Roswell Park Memorial Institute 1640 medium (Life Technologies, Carlsbad, CA, USA) supplemented with $10 \%$ fetal bovine serum (Hyclone, Logan, UT, USA). Cells were trypsinized and then stained with $0.2 \%$ trypan blue solution (Sigma, St Louis, MO, USA) and counted using a hemocytometer (Superior, Marienfeld, Germany).

\section{Western blotting analysis}

Cells were washed three times with phosphate-buffered saline (PBS) and lysed with lysis buffer containing $20 \mathrm{~mm}$ Tris- $\mathrm{HCl}$ ( $\mathrm{pH} 7.4$ ), $5 \mathrm{~mm}$ EDTA (pH 8.0), $10 \mathrm{~mm} \mathrm{Na}_{4} \mathrm{P}_{2} \mathrm{O}_{7}, 100 \mathrm{~mm} \mathrm{NaF}, 2 \mathrm{~mm} \mathrm{Na}_{3} \mathrm{VO}_{4}, 1 \%$ NP-40 and protease and phosphatase inhibitors. The protein concentration in the whole-cell lysate was determined using the BCA assay (Thermo Fisher Scientific, Waltham, MA, USA). Denatured protein $(25 \mu \mathrm{g})$ was separated on a $10 \%$ polyacrylamide gel and transferred to a polyvinylidene difluoride membrane. The membrane was blocked with 5\% skim milk in Tris-buffered saline (TBS) containing $0.1 \%$ Tween 20 (TBST) and incubated with each primary antibody overnight at $4{ }^{\circ} \mathrm{C}$. The primary antibody against ERR $\gamma$ was purchased from R\&D Systems; antibodies against p21 and p27 were purchased from BD Pharmingen (Lexington, KY, USA); the antibody against phosphorylated retinoblastoma protein ( $\mathrm{p}-\mathrm{pRb})$ was purchased from Cell Signaling Technology (Beverly, MA, USA); and the antibodies against total-pRb and $\beta$-actin were purchased from Santa Cruz Biotechnology (Santa Cruz, CA, USA). Membranes were washed three times with TBST and incubated with horseradish peroxidase-conjugated mouse (Santa Cruz) or rabbit (Cell Signaling Technology) secondary antibodies. ECL reagent (Bionote, Hwaseong-si, Korea) was used to detect horseradish peroxidase, and images were analyzed using an ImageQuant LAS4000 mini (GE healthcare Life Science, Piscataway, NJ, USA).

\section{Small interfering RNA (siRNA) transfection}

Cells were transfected with $50 \mathrm{~nm}$ of scrambled siRNA or siRNA-ERR $\gamma$ using lipofectamine LTX (Invitrogen, Carlsbad, CA, USA), according to the manufacturer's protocol. The following siRNA sequence was 
used for ERR $\gamma$ silencing: sense: 5'-CUGAUAGGUUUCCUCUACU-3', and antisense: 5'-AGUAGAGGAAACCUAUCAG-3'.

\section{Flow cytometric analysis}

The cell cycle was analyzed by staining cells with propidium iodide. Briefly, cells were trypsinized, washed twice with PBS and fixed with absolute EtOH overnight at $-20^{\circ} \mathrm{C}$. The fixed cells were then washed twice with PBS and stained with $0.5 \mu \mathrm{g} \mathrm{ml}^{-1}$ propidium iodide solution. The cell cycle was examined using an Accuri C6 flow cytometer (BD Biosciences, San Jose, CA, USA) operating at $585 / 40 \mathrm{~nm}$ with a fl2/A filter. After gating to eliminate debris and doublets using side scatter and forward scatter, $10^{4}$ cells were collected and analyzed per sample. Analysis was performed with the BD Accuri C6 software (BD Biosciences).

\section{DCF-DA staining}

The generation of reactive oxygen species (ROS) by ERR $\gamma$ inhibition was analyzed by chloromethyl-2', $7^{\prime}$-dichlorodihydrofluorescein diacetate (CM-H 2 DCF-DA) staining. Briefly, $20 \mu \mathrm{M}$ of DCF-DA was added to cells plated on a coverslip and incubated for $30 \mathrm{~min}$. Cells were then washed three times with PBS and fixed in $4 \%$ paraformaldehyde for $20 \mathrm{~min}$ at room temperature. Fixed cells were then washed three times with PBS, and images were captured using an Olympus IX81 fluorescence microscope (Olympus Corp., Tokyo, Japan).

\section{Luciferase reporter assay}

$\mathrm{PLC} / \mathrm{PRF} / 5$ cells were used for transient transfection assays. Cells were transfected with a luciferase reporter gene plasmid using Mirus TransIT-LT1 transfection reagent (Mirus Bio LLC, Madison, WI, USA), according to the manufacturer's protocol. Cells were transfected for 1 day and then treated with GSK5182 for 2 days. Luciferase activity was then measured using a luminometer (Berthold Detection Systems, Pforzheim, Germany). $\beta$-Galactosidase activity was used for normalization. The plasmid encoding stf4-Luc was kindly donated by HeungSik Choi (Chonnam National University, Gwangju, Korea). ${ }^{25}$

\section{Chemicals}

GSK5182 was synthesized as previously described. ${ }^{26}$ GSK5182 was obtained as an $\mathrm{HCl}$ salt, dissolved in dimethyl sulfoxide and used at the indicated concentrations for in vitro experiments. $\mathrm{N}$-acetylcysteine was purchased from Sigma.

\section{Relative quantification analysis}

Relative protein levels and fluorescence signals for DCA-DA were analyzed using the Image J software (US National Institutes of Health, Bethesda, MD, USA).

\section{Ethics statement}

The study was approved by the institutional review board (IRB) of Keimyung University Dongsan Hospital (IRB no: 11-256). Written informed consent was obtained from each patient.

\section{Statistical analyses}

Statistical analysis was performed using a two-tailed Student's $t$-test. Data are expressed as the mean \pm s.e.m. Clinical data management and statistical analyses were performed using the SPSS software (version 18.0; SPSS, Inc., Chicago, IL, USA). The association between protein levels and categorical variables was evaluated using Pearson's $\chi^{2}$ test. Overall survival was estimated using the Kaplan-Meier method. $P<0.05$ was considered statistically significant.

\section{RESULTS}

ERR $\gamma$ levels in HCC and their association with clinical stage and pathological grade

We first measured the level of ERR $\gamma$ in tumors obtained from 190 HCC patients after liver resection and compared it with that in adjacent non-tumor tissues obtained from the same patients. The baseline characteristics of the 190 patients $^{24}$ are described in Supplementary Table S1. The immunoreactivity of ERR $\gamma$ in non-tumor and tumor tissues was scored from 0 to $3+$ according to the intensity of staining. Immunohistochemical analysis revealed that 183 non-tumor lesions stained negative, with only four cases staining positive. By contrast, only 55 tumor lesions stained negative, whereas 135 cases stained positive. Notably, high levels of ERR $\gamma$ (defined as an immunoreactivity score of $2+$ or $3+$ ) were observed only in tumor tissues; all non-tumor tissues expressed low levels of ERR $\gamma$ (immunoreactivity score of 0 or $1+$ ) (Figure 1a).

We next examined whether this upregulation correlated with tumor stage and grade. The results showed that a higher percentage of patients with low-level ERR $\gamma$ expression had tumors at TNM stage I and II, whereas a higher percentage of patients with high-level ERR $\gamma$ expression had tumors at TNM stage III $(P=0.005$; Figure 1b). Furthermore, a significantly higher percentage of patients with low-level ERR $\gamma$ expression had early-stage BCLC ( 0 and A) and showed a lower Edmondson-Steiner grade (I and II) (Figures $1 \mathrm{c}$ and $\mathrm{d}$; $P=0.049$ and $P=0.026$, respectively). Taken together, these results suggest that the ERR $\gamma$ level was markedly higher in HCC than in adjacent non-tumor liver tissues and that higher ERR $\gamma$ immunoreactivity correlated with more advanced clinical stage and higher HCC pathological grade.

\section{Relationship between ERR $\gamma$ level and overall survival} Next we examined the relationship between ERR $\gamma$ immunoreactivity and overall survival. The median follow-up period was $31.4 \pm 1.41$ (range, 0.2-136.0) months. Although there was no correlation between ERR $\gamma$ level and survival at early TNM stages (stages I and II) $(P=0.200$, Figure 2a), high-level ERR $\gamma$ expression and an advanced TNM stage (stage III) correlated with shorter overall survival (median survival, 47.1 months vs 99.9 months for high and low level of ERR $\gamma$ immunoreactivity in tumors, respectively; $P=0.012$; Figure 2b).

\section{Knocking down ERR $\gamma$ inhibits HCC cell proliferation and induces G1-phase arrest}

Because ERR $\gamma$ was upregulated in human HCC tissue, we next explored whether downregulating ERR $\gamma$ inhibits HCC cell proliferation and growth. As shown in Figure 3a, siRNAmediated knockdown of ERR $\gamma$ inhibited PLC/PRF/5 cell proliferation. We then examined whether knocking down ERR $\gamma$ inhibits cell growth by measuring the expression of cell cycle regulatory proteins and by fluorescence-activated cell sorting analysis of cell cycle progression. Immunoblot analysis showed that siRNA-ERR $\gamma$ increased the expression of the cyclin-dependent kinase inhibitors, p21 and p27, but reduced 
a
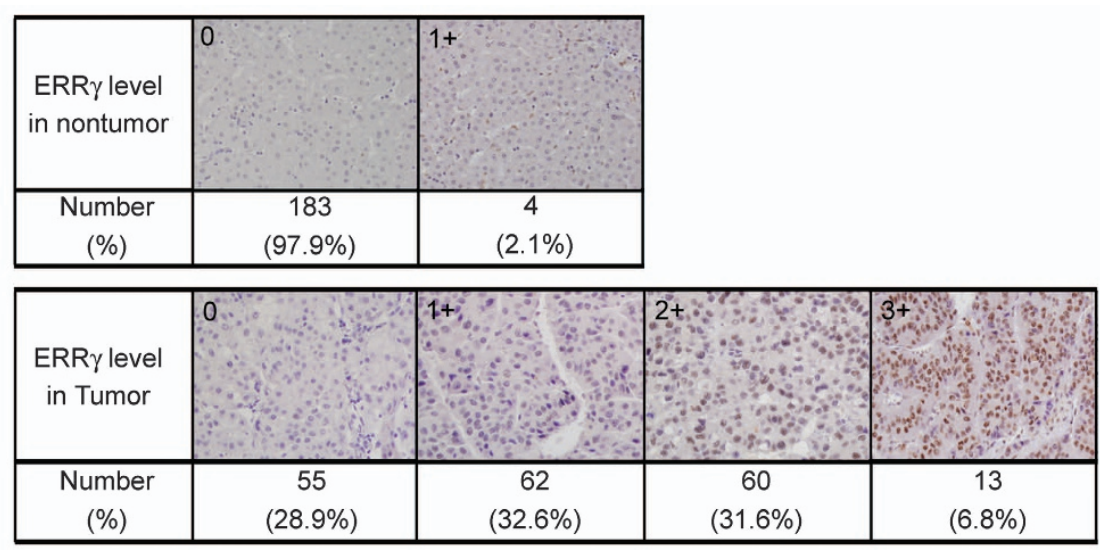

b

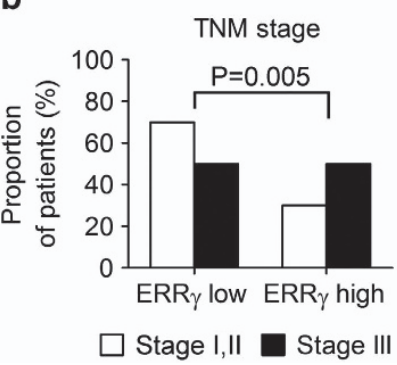

C
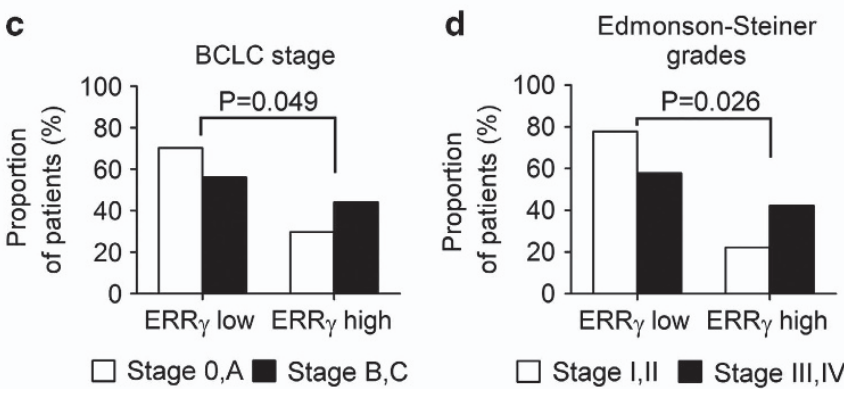

Figure 1 Immunohistochemical (IHC) analysis of ERR expression in surgically resected human HCC tumors and corresponding adjacent non-tumor tissues and its clinicopathological associations. (a) Representative images showing IHC staining of ERR $\gamma$ in tumor ( $n=190)$ and adjacent non-tumor $(n=187$ ) lesions (original magnification: $\times 400)$. The degree of IHC reactivity was scored from 0 to 3 according to the proportion of positively stained tumor cells: 0 , none; $1+,<10 \% ; 2+, 10-25 \% ; 3+,>25 \%$. Three cases of non-tumor tissues were missed. (b-d) Proportion of tumors with early and advanced TNM (b) and BCLC (c) stages and with low and high Edmondson grades (d) according to ERR $\gamma$ expression.

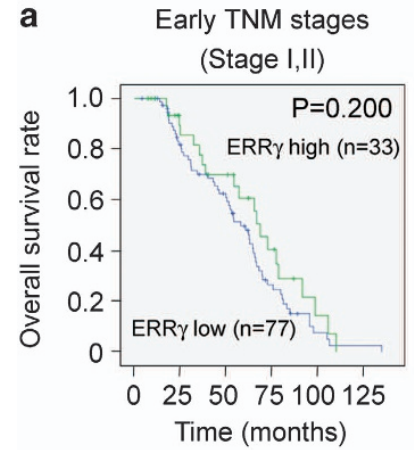

b Advanced TNM stage

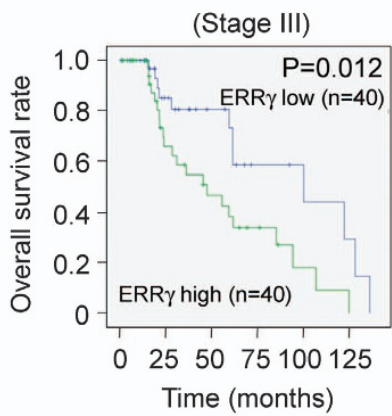

- ERR $\gamma$ low group

- ERR $\gamma$ high group

Figure 2 Kaplan-Meier analysis of the survival of HCC patients according to ERR $\gamma$ immunoreactivity. (a) Early TNM stages (I and II). (b) advanced TNM stage (III). Low-level ERR $\gamma$ expression was defined as a score of 0 or $1+$; high-level ERR $\gamma$ expression was defined as a score of $2+$ or $3+$.

p-pRb levels (Figure 3b). Flow cytometric analysis revealed that siRNA-ERR $\gamma$ caused a significant increase in the percentage of cells in G1 phase $(55.2 \%$ in transfected cells vs $38.2 \%$ in control cells), with a concomitant decrease in the percentage of cells in S phase $(17.7 \%$ in transfected cells vs $26.3 \%$ in control cells) (Figure 3c).
The ERR $\gamma$ inverse agonist, GSK5182, attenuates HCC cell proliferation

Next we used the inverse agonist, GSK5182, to examine whether pharmacological inhibition of ERR $\gamma$ activity has an antitumor effect. HCC cells were transiently transfected with the Stf4 luciferase reporter plasmid containing the ERR $\gamma$ response element and then exposed to various doses of GSK5182. ${ }^{25}$ A luciferase reporter assay revealed that GSK5182 significantly attenuated ERR $\gamma$ activity in a dose-dependent manner (Figure 4a). Consistent with the results from experiments involving endogenous knockdown of ERR $\gamma$, we found that treatment with GSK5182 led to a significant and dosedependent reduction in the number of proliferating PLC/PRF/5 cells (Figure 4b). GSK5182 also caused a dose-dependent increase in the expression of p21 and p27 while at the same time reducing the level of p-pRb (Figure 4c). The results obtained from flow cytometry revealed that GSK5182 induced cell cycle arrest at G1 phase, which in turn induced a corresponding dose-dependent reduction in the percentage of cells in $S$ phase (Figure $4 \mathrm{~d}$ ). Taken together, these data indicate that the antitumor effect of GSK5182 in HCC cells is mediated by the induction of cell cycle arrest at the G1/S checkpoint.

The antitumor effects of GSK5182 are also mediated by ROS To examine the factors that contribute to GSK5182-induced cell cycle arrest, we next examined whether cellular GSK5182 

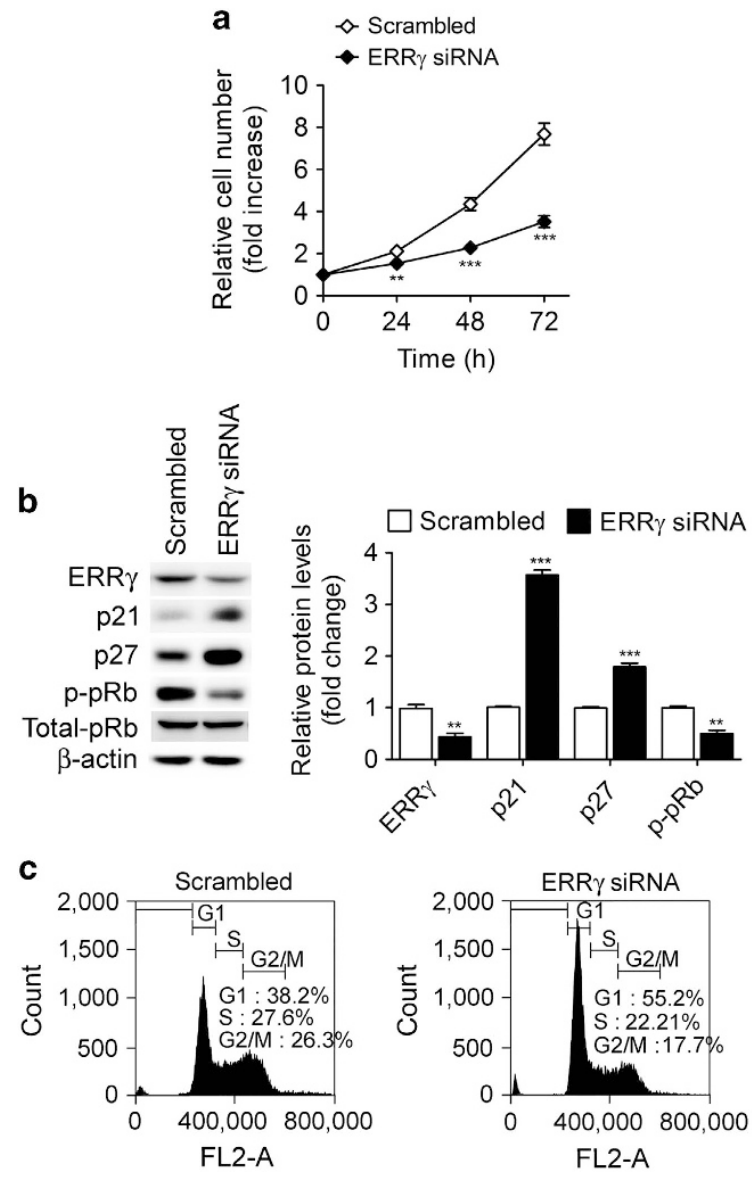

Figure 3 Knocking down ERR $\gamma$ inhibits $\mathrm{HCC}$ cell proliferation and induces G1 phase arrest. (a) Growth curve analysis of PLC/PRF/5 cells after transfection with scrambled or siRNA-ERR $\gamma$. A white circle denotes scrambled siRNA and a black circle denotes ERR $\gamma$ SiRNA. (b) Representative western blotting showing the effect of siRNA-ERR $\gamma$ on the levels of cell cycle regulatory proteins. PLC/ $\mathrm{PRF} / 5$ cells were transfected with scrambled siRNA or siRNA-ERR $\gamma$ for 2 days. ERR $\gamma, p 21$ and p27 levels were normalized to $\beta$-actin and phospho-pRb levels were normalized to total-pRb levels. Data are expressed as the mean \pm s.e.m. of three independent measurements. ${ }^{* *} P<0.01$, and ${ }^{* * *} P<0.001$ vs scrambled control. (c) Representative flow cytometric data derived from the analysis of cell cycle progression in PLC/PRF/5 cells. Quiescent cells were transfected with scrambled siRNA or siRNA-ERR $\gamma$. Propidium iodide-stained cells were separated by flow cytometry.

increased ROS levels. As shown in Figure 5a, GSK5182 increased the level of cellular ROS (as measured by DCF-DA staining) in a dose-dependent manner (Figure 5a). Moreover, pretreatment with the antioxidant, $\mathrm{N}$-acetylcysteine, attenuated GSK5182-induced suppression of HCC cell proliferation (Figure 5b), indicating that the antitumor effects of GSK5182 are, at least in part, mediated via ROS generation.

\section{DISCUSSION}

Here we showed that the level of ERR $\gamma$-positivity in HCC tissues was markedly higher than that in adjacent non-tumor liver tissues and that tumors expressing high levels of ERR $\gamma$ were of more advanced clinical stage and pathological grade.
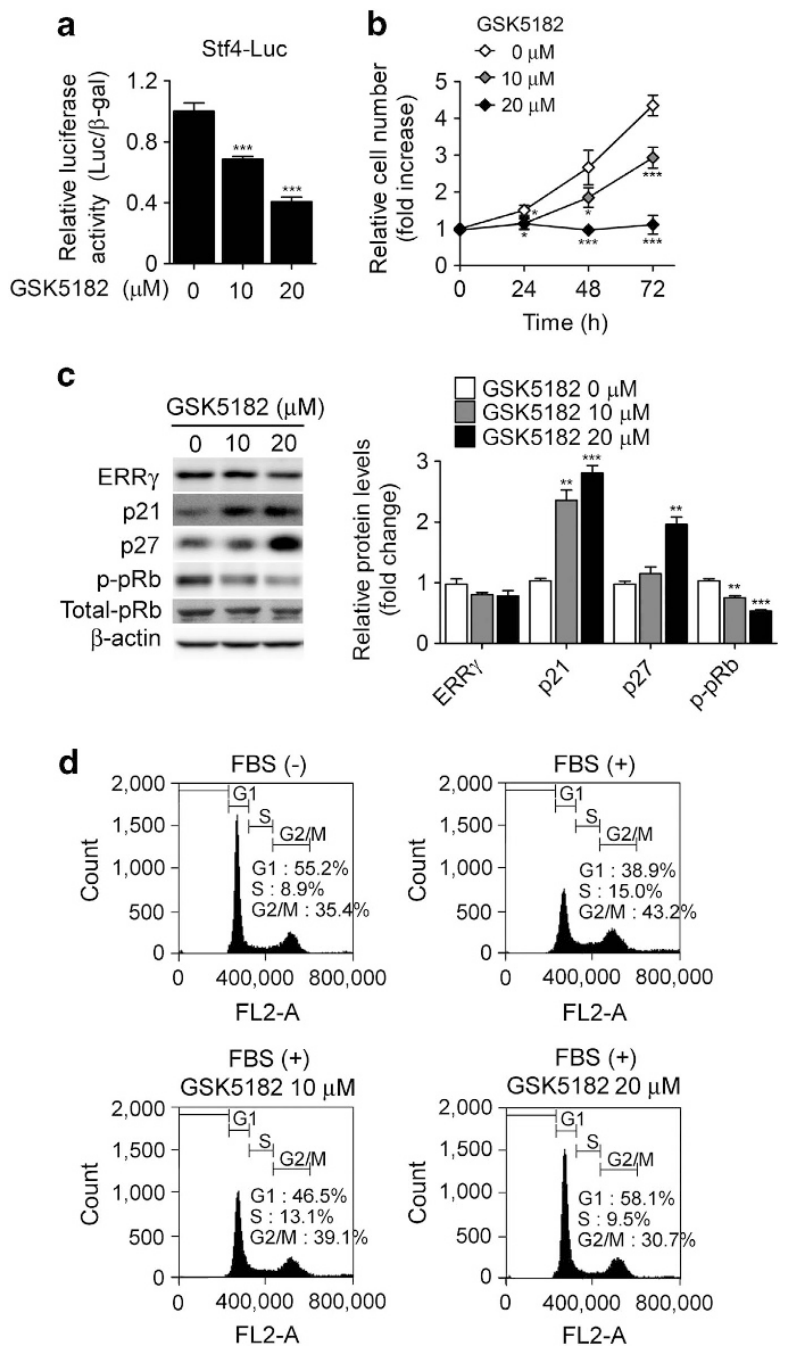

Figure 4 An ERR inverse agonist, GSK5182, inhibits HCC cell proliferation. (a) Luciferase reporter assays were used to examine the effect of GSK5182, which inhibits ERR $\gamma$ activity. PLC/PRF/5 cells were transfected with the luciferase reporter construct stf4Luc in the presence of the indicated doses of GSK5182. Relative promoter activity was determined after 2 days. (b) Growth curve analysis of HCC cells after treatment with DMSO or with the indicated doses of GSK5182. A white circle denotes the vehicle; a gray circle denotes $10 \mu \mathrm{m}$ GSK5182; a black circle denotes $20 \mu \mathrm{m}$ GSK5182. (c) Representative western blotting showing the effect of GSK5182 on the expression of cell cycle regulatory proteins. PLC/ $\mathrm{PRF} / 5$ cells were treated with DMSO or with the indicated doses of GSK5182 for $24 \mathrm{~h}$. ERR $\gamma$, p21 and p27 levels were normalized to $\beta$-actin and phospho-pRb levels were normalized to total-pRb levels. Data are expressed as the mean \pm s.e.m. of three independent measurements. ${ }^{*} P<0.05,{ }^{* *} P<0.01$ and ${ }^{* * *} P<0.001$ vs control. (d) Representative flow cytometric data derived from analysis of cell cycle progression in PLC/PRF/5 cells. Quiescent cells were treated with DMSO or with the indicated doses of GSK5182. Propidium iodide-stained cells were separated by flow cytometry.

Furthermore, we found that inhibiting ERR $\gamma$ had an apparent therapeutic effect on HCC cells by inducing cell cycle arrest.

Previous studies have noted inconsistent expression of ERR $\gamma$ in various tumor tissues and have attempted to examine its role 


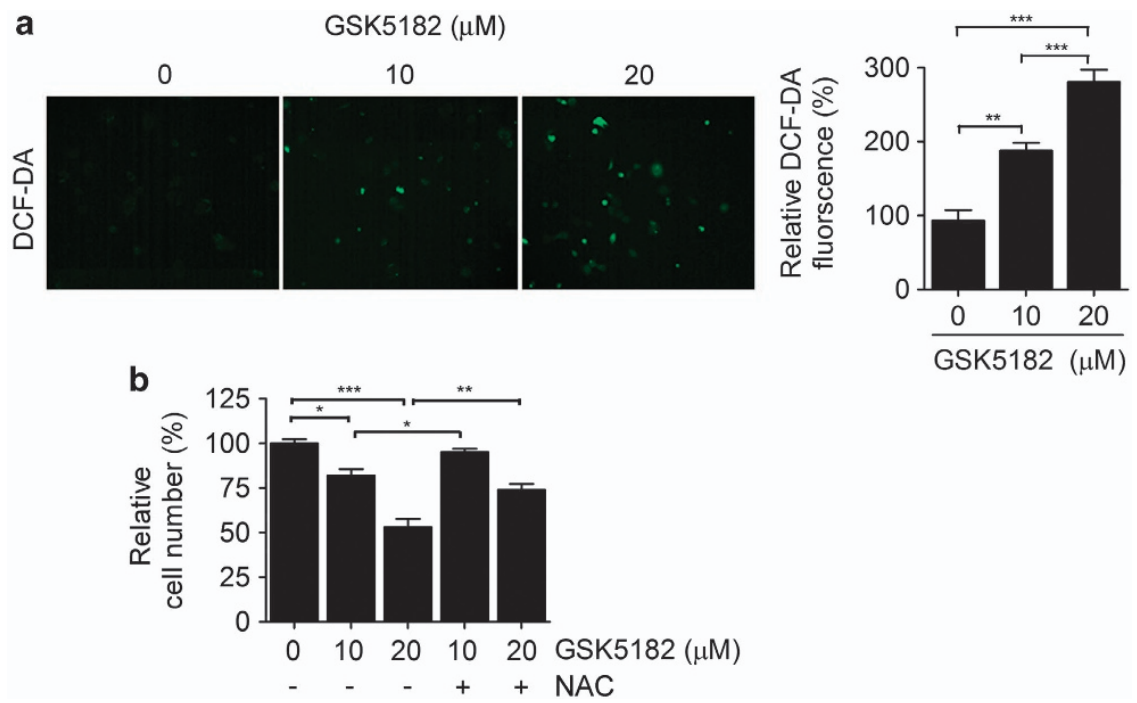

Figure 5 GSK5182 induces ROS generation and NAC attenuates GSK5182-induced suppression of HCC cell proliferation. (a) Representative DCF-DA fluorescence images showing the effect of GSK5182 on ROS generation in PLC/PRF/5 cells. Cells were treated with DMSO or with the indicated doses of GSK5182 for $24 \mathrm{~h}$ and then stained with 2', 7'-dichlorodihydrofluorescein diacetate (DCF-DA). (Original magnification: $\times 200$ ). (b) Effect of NAC on GSK5182-induced inhibition of HCC cell proliferation. PLC/PRF/5 cells were pretreated (or not) with $8 \mathrm{~mm}$ NAC for $1 \mathrm{~h}$, followed by incubation with the indicated doses of GSK5182 for $24 \mathrm{~h}$. Data are expressed as the mean \pm s.e.m. of three independent measurements. ${ }^{*} P<0.05,{ }^{*} P<0.01$ and ${ }^{* * *} P<0.001$ vs indicated group.

in tumor growth. ${ }^{8,9,19}$ Overexpression of ERR $\gamma$ is frequently observed in endometrial and breast cancers, whereas ERR $\gamma$ is downregulated in many prostate cancer cell lines; this suggests that ERR $\gamma$ has different roles depending on the tumor type. ${ }^{19}$ The present study showed that ERR $\gamma$ was upregulated in human HCC tissue but was rarely expressed in adjacent normal liver tissue. The clinical significance of high ERR $\gamma$ expression in patients is highlighted by the results showing that tumors expressing high levels of ERR $\gamma$ were also of advanced TNM and BCLC stage and had a higher Edmondson-Steiner grade. We also showed that high levels of ERR $\gamma$ expression in patients with TNM stage III tumors were associated with worse overall survival, supporting the role of ERR $\gamma$ as a potential prognostic marker for advanced HCC. However, because the study cohort included patients who had undergone curative tumor resection, none of the patients had TNM stage IV tumors. Therefore, to fully elucidate the clinical significance of ERR $\gamma$ at all stages of HCC, further studies should examine the association between $\mathrm{ERR} \gamma$ and clinical features in a cohort that includes patients with inoperable tumors. Considering that early detection of small HCC is an important prognostic factor for curative resection, it is also important to identify biomarkers that increase diagnostic accuracy. Although the cohort in the present study was not selected to estimate diagnostic accuracy, the tumor-specific expression of ERR $\gamma$ suggests that it is of diagnostic value. Indeed, the sensitivity, specificity and positive and negative predictive value of ERR $\gamma$ for HCC were 71, 98, 97 and $77 \%$, respectively. These results are comparable with those obtained for other proven HCC markers. ${ }^{27-29}$ Taken together, the results presented herein suggest that ERR $\gamma$ might be a novel prognostic marker of an unfavorable clinical outcome and may have utility as a diagnostic biomolecule that can improve diagnostic accuracy for HCC when combined with other tissue markers.

The association between ERR $\gamma$ expression in HCC tissue and clinical significance prompted us to investigate the possibility that ERR $\gamma$ may be a potential therapeutic target for HCC. We examined this possibility using siRNA-mediated knockdown of ERR $\gamma$ and a 4-hydroxy tamoxifen analog, GSK5182, which is a selective inverse agonist of ERR $\gamma$ that does not interact with other nuclear receptors, including $\mathrm{ERR} \alpha$ or $\mathrm{ER} \alpha$, owing to additional noncovalent interactions with Y326 and N346 within the active site of ERR $\gamma .{ }^{26,30}$ Indeed, ERR $\gamma$ knockdown and GSK5182 treatment reduced HCC cell proliferation by inducing cell cycle arrest. The results showing that ERR $\gamma$ knockdown and GSK5182 treatment upregulated p21 and p27 protein levels suggest that the antitumor effects occur via p21- and p27-mediated blockade of G1-S transition. Our data are consistent with a previous study showing that overexpression of ERR $\gamma$ induced S-phase cell cycle progression in Hela cells through the induction of $P l k 2$ expression. ${ }^{31}$ Mounting evidence shows that p21-mediated oxidative stress induces p53-dependent and p53-independnt cell cycle arrest. $^{14}$ In line with this, our own results showed that GSK5182 induces ROS generation and that antioxidant treatment prevents the GSK5182-induced reduction of HCC cell proliferation, indicating that GSK5182 mediated its effects via ROS generation.

To the best of our knowledge, the present study is the first to show the clinical significance of ERR $\gamma$ in HCC. The evidence presented herein strongly suggests that further studies are necessary to fully elucidate whether ERR $\gamma$ is a novel biomarker for the diagnosis and prognosis of HCC. Furthermore, the finding that GSK5182 inhibits of HCC 
growth and proliferation raises the possibility that chemical inhibitors of ERR $\gamma$ may be a potential resource for HCC treatment.

\section{CONFLICT OF INTEREST}

The authors declare no conflict of interest.

\section{ACKNOWLEDGEMENTS}

This work was supported by grants (NRF-2015R1A2A1A15053422 and NRF-2015R1A2A1A10052745) the National Research Foundation of Korea funded by the Ministry of Science, ICT and Future Planning, and grants (HI11C1300, HI15C0001 and HI13C1905) from the Korea Health technology R\&D Project through the Korea Health Industry Development Institute (KHIDI), funded by the Ministry of Health \& Welfare, Republic of Korea).

1 Ferlay J, Shin HR, Bray F, Forman D, Mathers C, Parkin DM. Estimates of worldwide burden of cancer in 2008: GLOBOCAN 2008. Int J Cancer 2010; 127: 2893-2917.

2 Bruix J, Sherman M. Management of hepatocellular carcinoma: an update. Hepatology 2011; 53: 1020-1022.

3 Llovet JM, Bruix J. Molecular targeted therapies in hepatocellular carcinoma. Hepatology 2008; 48: 1312-1327.

4 Razzaque MA, Masuda N, Maeda Y, Endo Y, Tsukamoto T, Osumi T. Estrogen receptor-related receptor gamma has an exceptionally broad specificity of DNA sequence recognition. Gene 2004; 340: 275-282.

5 Giguere V. To ERR in the estrogen pathway. Trends Endocrinol Metab 2002; 13: 220-225.

6 Bookout AL, Jeong Y, Downes M, Yu RT, Evans RM, Mangelsdorf DJ. Anatomical profiling of nuclear receptor expression reveals a hierarchical transcriptional network. Cell 2006; 126: 789-799.

7 Giguere V. Transcriptional control of energy homeostasis by the estrogenrelated receptors. Endocr Rev 2008; 29: 677-696.

8 Riggins RB, Lan JP, Zhu Y, Klimach U, Zwart A, Cavalli LR et al. ERRgamma mediates tamoxifen resistance in novel models of invasive lobular breast cancer. Cancer Res 2008; 68: 8908-8917.

9 Heckler MM, Thakor H, Schafer CC, Riggins RB. ERK/MAPK regulates ERRgamma expression, transcriptional activity and receptor-mediated tamoxifen resistance in ER+ breast cancer. FEBS J 2014; 281: 2431-2442.

10 Ren Z, Yang H, Wang C, Ma X. The effects of PGC-1alpha on the proliferation and energy metabolism of malignant endometrial cancer cells. Onco Targets Ther 2015; 8: 769-774.

11 Sun $\mathrm{Y}$, Wang C, Yang H, MaX. The effect of estrogen on the proliferation of endometrial cancer cells is mediated by ERRgamma through AKT and ERK1/2. Eur J Cancer Prev 2014; 23: 418-424.

12 Lapenna S, Giordano A. Cell cycle kinases as therapeutic targets for cancer. Nat Rev Drug Discov 2009; 8: 547-566.

13 Waldman T, Kinzler KW, Vogelstein B. p21 is necessary for the p53-mediated G1 arrest in human cancer cells. Cancer Res 1995; 55: 5187-5190.

14 Gartel AL, Tyner AL. The role of the cyclin-dependent kinase inhibitor p21 in apoptosis. Mol Cancer Ther 2002; 1: 639-649.

15 Senderowicz AM. Small-molecule cyclin-dependent kinase modulators. Oncogene 2003; 22: 6609-6620.

16 Bianco S, Sailland J, Vanacker JM. ERRs and cancers: effects on metabolism and on proliferation and migration capacities. J Steroid Biochem Mol Biol 2012; 130: 180-185.
17 Deblois G, Hall JA, Perry MC, Laganiere J, Ghahremani M, Park M et al. Genome-wide identification of direct target genes implicates estrogenrelated receptor alpha as a determinant of breast cancer heterogeneity. Cancer Res 2009; 69: 6149-6157.

18 Bernatchez G, Giroux V, Lassalle T, Carpentier AC, Rivard N, Carrier JC. ERRalpha metabolic nuclear receptor controls growth of colon cancer cells. Carcinogenesis 2013; 34: 2253-2261.

19 Cheung CP, Yu S, Wong KB, Chan LW, Lai FM, Wang X et al. Expression and functional study of estrogen receptor-related receptors in human prostatic cells and tissues. J Clin Endocrinol Metab 2005; 90: $1830-1844$

20 Yu S, Wong YC, Wang XH, Ling MT, Ng CF, Chen S et al. Orphan nuclear receptor estrogen-related receptor-beta suppresses in vitro and in vivo growth of prostate cancer cells via p21(WAF1/CIP1) induction and as a potential therapeutic target in prostate cancer. Oncogene 2008; 27: 3313-3328.

21 Bianco S, Lanvin O, Tribollet V, Macari C, North S, Vanacker JM. Modulating estrogen receptor-related receptor-alpha activity inhibits cell proliferation. J Biol Chem 2009; 284: 23286-23292.

22 Edge SB, Compton CC. The American Joint Committee on Cancer: the 7th edition of the AJCC cancer staging manual and the future of TNM. Ann Surg Oncol 2010; 17: 1471-1474.

23 Llovet JM, Bru C, Bruix J. Prognosis of hepatocellular carcinoma: the BCLC staging classification. Semin Liver Dis 1999; 19: 329-338.

24 Lee YJ, Hah YJ, Kang YN, Kang KJ, Hwang JS, Chung WJ et al. The autophagy-related marker LC3 can predict prognosis in human hepatocellular carcinoma. PLOS ONE 2013; 8: e81540.

25 Sanyal S, Kim JY, Kim HJ, Takeda J, Lee YK, Moore DD et al. Differential regulation of the orphan nuclear receptor small heterodimer partner (SHP) gene promoter by orphan nuclear receptor ERR isoforms. J Biol Chem 2002; 277: 1739-1748.

26 Chao EY, Collins JL, Gaillard S, Miller AB, Wang L, Orband-Miller LA et al. Structure-guided synthesis of tamoxifen analogs with improved selectivity for the orphan ERRgamma. Bioorg Med Chem Lett 2006; 16: 821-824.

27 Tremosini S, Forner A, Boix L, Vilana R, Bianchi L, Reig M et al. Prospective validation of an immunohistochemical panel (glypican 3, heat shock protein 70 and glutamine synthetase) in liver biopsies for diagnosis of very early hepatocellular carcinoma. Gut 2012; 61: 1481-1487.

28 Di Tommaso L, Franchi G, Park YN, Fiamengo B, Destro A, Morenghi E et al. Diagnostic value of HSP70, glypican 3, and glutamine synthetase in hepatocellular nodules in cirrhosis. Hepatology 2007; 45: 725-734.

29 International Consensus Group for Hepatocellular Neoplasia. Pathologic diagnosis of early hepatocellular carcinoma: a report of the International Consensus Group for Hepatocellular Neoplasia. Hepatology 2009; 49. 658-664.

30 Kim DK, Ryu D, Koh M, Lee MW, Lim D, Kim MJ et al. Orphan nuclear receptor estrogen-related receptor gamma (ERRgamma) is key regulator of hepatic gluconeogenesis. J Biol Chem 2012; 287: 21628-21639.

31 Park YY, Kim SH, Kim YJ, Kim SY, Lee TH, Lee IK et al. Polo-like kinase 2 gene expression is regulated by the orphan nuclear receptor estrogen receptor-related receptor gamma (ERRgamma). Biochem Biophys Res Commun 2007; 362: 107-113.

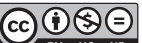

This work is licensed under a Creative Commons Attribution-NonCommercial-NoDerivs 4.0 International License. The images or other third party material in this article are included in the article's Creative Commons license, unless indicated otherwise in the credit line; if the material is not included under the Creative Commons license, users will need to obtain permission from the license holder to reproduce the material. To view a copy of this license, visit http://creativecommons.org/licenses/by-nc-nd/4.0/

Supplementary Information accompanies the paper on Experimental \& Molecular Medicine website (http://www.nature.com/emm) 